\title{
Near peer teaching in general practice: option or expectation?
}

\author{
Hugh Alberti ${ }^{1}$, Joe Rosenthal ${ }^{2}$, Liza Kirtchuk ${ }^{3}$, Harish Thampy ${ }^{4}$, Michael Harrison ${ }^{1}$ \\ ${ }^{1}$ School of Medical Education, Faculty of Medical Sciences, Newcastle University \\ ${ }^{2}$ University College London Research Department of Primary Care and Population Health, \\ London \\ ${ }^{3}$ School of Population Sciences and Health Services Research, King's College London School \\ of Medicine, London \\ ${ }^{4}$ Division of Medical Education, University of Manchester
}

Corresponding author:

Michael Harrison

e-mail: Mike.Harrison@newcastle.ac.uk

tel: 01912087005 


\title{
Near peer teaching in general practice: option or expectation?
}

\author{
Abstract \\ GP trainees who teach medical students do so as near peers with established educational \\ benefits for all concerned. Through teaching, GP trainees consolidate their own knowledge \\ and skills whilst students value the experience of learning from teachers closer in age and \\ stage. Importantly, involving GP trainees as teachers also increases primary care teaching \\ capacity and promotes general practice as a potential career option for undergraduates. \\ However, whilst junior doctors are often to be found teaching on hospital wards and in \\ clinics, GP trainees based in primary care appear to have fewer opportunities to teach. This \\ article encourages the promotion of near peer teaching in primary care on a number of levels. \\ We make practical suggestions that may be of benefit to the individual GP trainee, trainer and \\ practice; and also discuss ways in which key stakeholders, including medical schools and \\ those responsible for organising post-graduate primary care training programmes, may \\ promote near peer teaching in general practice. We propose that all medical students should \\ have experience of being taught by GP trainees, and likewise that all future GPs should have \\ training and experience of teaching undergraduate medical students.
}

\section{Keywords}

Near peer teaching, vertical integration, community-based education, general practice speciality training, post-graduate medical education

\section{Introduction}

The value and potential of near peer teaching in primary care medical education has been increasingly recognised in several countries in recent years (1). Reasons for this include 
growth in medical student numbers, demand for more high quality placements, and increasing shift of health care provision from secondary care to the community. There is also increasing international recognition of the important role that doctors in training play in the education and development of medical students (2). Through increasing opportunities to interact with medical students, trainees can influence students' career choice $(3,4)$ and improve students' perceptions of the specialty $(5,6)$.

Provision of the teaching and support required for these increasing numbers of students has been a challenge for medical schools. We have argued elsewhere that part of the answer in the UK should be greater involvement of general practice (GP) trainees who represent a large, well-placed but under-utilised educational resource (1). The UK General Medical Council (GMC) states that all doctors should be willing and able to contribute to teaching activities (7). Similarly, one of the Royal College of General Practitioners (RCGP) curriculum outcomes for GP trainees states that 'in the latter stages of training GP trainees are ideally placed to deliver some of the teaching, mentoring and clinical supervision required'(8, p.4). The Health Education England and Medical Schools Council's report "By choice not by chance" specifically recommends that positive and enthusiastic general practitioner role models, such as GP trainees, should be identified and made visible across all medical schools and similar initiatives are underway internationally (9). However, it appears GP trainees are currently much less involved in teaching activities as compared to their hospital peers $(10,11)$ and reasons for this have been explored (11-14).

We believe it is timely to provide recommendations for all teachers, trainers and other stakeholders in primary care in order to capture the potential benefits of near peer teaching both for GP trainees and for medical students on primary care placements. The following recommendations emerge from the literature, our own experience, and ideas provided by the UK GP Heads of Teaching group. Our recommendations are aimed at various levels from the 
individual trainee to the trainers, training practices and those responsible for designing and delivering primary care training programmes.

\section{Trainee-level recommendations}

All GP trainees should be encouraged to seek out teaching opportunities in both primary care and hospital-based posts. In the hospital, junior doctors contribute significantly to medical student teaching (15), and many GP trainees will have been involved in undergraduate teaching during their hospital placements. However, GP trainees appear to be less involved in teaching when on primary care placements compared to their hospital-based rotations.

Studies suggest that GP trainees are keen to be involved in teaching activities when on placement in primary care and those who do teach report that this enhances their training (10, 16). However, some trainees do have reservations about being involved in teaching in primary care, including finding time available due to competing clinical and educational priorities, and negative views from some GP trainers regarding their requirement to be involved in teaching activities (13). Trainees can help by taking a pro-active role in seeking teaching opportunities within primary care posts. General practice provides a rich learning environment, with numerous opportunities for teaching, including both classroom-based activities such as tutorials, and clinical teaching such as "parallel surgeries". Trainee involvement in teaching can be facilitated by their GP trainers, who are able to inform them of the local opportunities available for teaching during a placement planning meeting, and encourage trainees to consider the ways in which teaching may also help address their own educational and clinical learning needs, e.g. choosing to teach a topic on a weak clinical area. This encourages the trainee to take responsibility for their own learning, as well as ensuring teaching activities remain relevant to the trainee's own learning needs. 
GMC recommendations and the RCGP curriculum in the UK encourage all trainees to gain competences in teaching $(7,8,17)$, yet current assessment processes require no evidence of teaching experience or competence. We recommend that a minimum experience of teaching delivery should become a formal requirement for all GP trainees and that this should be included as a workplace-based assessment within their training portfolio. We would go so far as to argue that Health Education England (or equivalent postgraduate training authorities) should make it a requirement that in order to host postgraduate trainees all training practices should be active at some level in undergraduate teaching. This would be a positive step towards developing practices as flexible and continuous learning environments supporting undergraduate through foundation to postgraduate training.

\section{Practice-level recommendations}

Training practices hosting GP trainees are in a strong position to provide opportunities for trainees to teach undergraduates, and can do so by both providing access to learners, and supporting the timetabling of trainee teaching activity. Commonly perceived barriers to practices hosting undergraduate medical students include financial and service provision considerations. However, these matters can be mitigated if the teaching is shared with the GP trainee, whose own development is simultaneously addressed.

GP trainees can play an important role in more integrated educational approaches, such as the Vertical Integration (VI) model, which has become increasingly widespread in Australian primary healthcare settings (18). In this model there is a sharing of teaching and learning roles across the learner stages, leading to engagement in synergistic multi-level learning and teaching activities (19). GP trainees, foundation doctors (pre-vocational) and medical students of differing grades of seniority can engage in various cross-pollinating learning and 
teaching activities within the practice, with the overarching supervision of a senior GP. This approach acknowledges the complexities of educational systems and promotes a culture of lifelong learning, and the integration of learners, resources, objectives and activities allows for enhanced educational outcomes.

Despite concerns over resources and the impact on service provision, potential benefits of embedding a rich teaching culture within the practice include prestige associated with teaching, improved staff morale (20), workforce recruitment and retention and improved clinical indicators (21).

\section{Training programme and HEE-level recommendations}

Training programmes can facilitate opportunities and training for their trainees as teachers in a number of ways. For example, a local postgraduate training programme may wish to address the development of teaching skills amongst their cohort of trainees. Clinicians who teach often do so with little or no formal training and instead build up skills experientially (22). However, it is well recognised that teaching, rather than being an innate skill, is a skill to be acquired and developed over time. A recent survey of UK GP trainees found that although $62 \%$ were regularly involved with teaching, only $41 \%$ had ever received any training in this area with $92 \%$ of trainees expressing a desire for specific teaching-skills training (23). Course content of a "how to teach" course for GP trainees could include a basic foundation of educational theory and allow participants to learn and practice teaching skills applicable to primary-care settings such as clinical supervision, assessment of learners, provision of feedback and recognising learners with difficulties (2). The literature is rich with descriptions and evaluations of a variety of trainee-focussed "teach the teacher" courses ranging from single workshops to longitudinally integrated curricula (24). Common to them 
all however is a focus on encouraging trainees to understand the benefits both to them and to learners through teaching activities in order to create onward engagement. Furthermore it is increasingly recognised that teaching programmes should aim beyond skill acquisition and additionally incorporate activities that encourage trainee doctors to explore their values, motivations and sense of what it means to be a teacher (25).

Some GP training programmes have established a close relationship with their local medical school in order to provide integrated clinical academic training programmes. For example, Academic Clinical Fellowship (ACF) programmes may focus on research and teaching in combination with clinical training. We recommend that all such academic training programmes should include regular opportunities for trainees to teach undergraduates. Another example of medical schools and training practices working together is in the development of Integrated or Innovative Training Posts (ITPs) in medical education, which allow trainees to split their time between clinical general practice and undergraduate teaching (26). These posts provide trainees with protected time to teach, and ideally to complete an academic qualification in medical education. Such links also provide trainees with the opportunity to develop skills in medical education related research, as well as providing trainees with greater awareness of clinical academic career pathways. Evaluation of these posts demonstrates that GP trainee value these opportunities as they help to build their professional identity as educators as well as future GPs (27).

Health Education England, or equivalent postgraduate teaching bodies, may also be able to explore opportunities to fund teaching fellow posts with the remit of strengthening institutional links and increasing the teaching taking place in General Practice. These relationships will also allow for more multi-disciplinary approaches to education and training and may allow for trainees to undertake teaching of allied healthcare professionals. 


\section{Medical school-level recommendations}

Most GP trainees are keen to teach but they do need to be asked, encouraged and supported. Many have previous teaching experience, and all have knowledge and skills to share with medical students. Medical school undergraduate teaching leads should proactively make contact with local GP training programme directors for initial discussions on how to facilitate opportunities for their trainees to teach. For example, they could request an opportunity to discuss teaching opportunities within the trainees' half-day release programme or meet local GP trainers at one of their regular trainers' workshop meetings. Clear messages need to be provided at every opportunity as to the benefits of near peer teaching for students, trainees and practices, using examples of how teaching can be incorporated within clinical placements, what course materials are provided, what training and support is available for teachers and how funding works for the practice.

Ideally, trainees should have opportunities for hands-on experience of teaching students within their training practice. However, not all GP trainees will have the opportunity to teach within their training practice if the practice does not host medical students, although we would strongly encourage all training practices to host medical students. As a result, it is important that external opportunities are available that GP trainees can choose to participate in. Medical school-based activities might include delivering clinical skills, communication skills, facilitating small-group learning sessions and taking part in student assessments such as OSCEs. Medical schools should also consider utilising GP trainees in components of curricula that would particularly benefit from GP trainee in a broad range of curricular areas in light of both their generalist background and in the interests of encouraging good role modelling of primary and secondary care teams working together through curriculum 
delivery. Some of these formalised opportunities could be delivered jointly with experienced tutors, allowing the opportunity for trainees' teaching to be observed and feedback provided (28). Alternatively, local GP practices could be grouped together in a hub model to deliver teaching, which would expand opportunities for GP trainees who do not have students at their particular practice. It is important that trainees are provided with learner evaluation and feedback following any formalised teaching sessions they conduct in order to promote selfreflective development on these activities. GP trainers should be encouraged to discuss and review with their trainee their development as a teacher as they gain increasing experience and skills.

\section{Broader recommendations}

In the hospital, junior doctors and trainees' involvement in teaching is considered a normal part of their everyday practice. Conversely, in a primary care environment, the role of the GP trainee as a teacher appears to be far less normalised (14). Postgraduate GP training has traditionally prided itself on a one-to-one relationship between GP trainer and trainee. Although this has been seen as the gold standard in post-graduate medical education, this may have helped promote a hierarchical structure of roles within training practices, in which the role of the trainee in primary care is seen as a learner only, whose priority is their own personal clinical and educational needs. This may contribute to forming a culture in primary care in which teaching is viewed as an extraneous rather than integral part of GP training. Similarly, culture and attitudes towards teaching within an individual GP practice may influence whether a GP trainee becomes involved in teaching activities.

Attempts to create a cultural shift in which this role is normalised are vital if there is to be an increased level of involvement of GP trainees in teaching activities in primary care. There 
needs to be a greater visible presence of GP trainee teachers in the primary care environment. This may be achieved through the development of flexible GP training posts, initiatives by training programmes to allow trainees time out of practice to teach, and education of GP trainers regarding how they may be able to involve trainees in primary care teaching. There also need to be efforts by key stakeholder institutions nationally and regionally, such as the RCGP and regional training programmes, to promote the role of the GP trainee as teacher.

There remains an ongoing discrepancy between the involvement of hospital and GP based trainees in teaching medical students. Whilst hospital-trainee delivered teaching is routine and normalised within secondary care settings, this is not the case in primary care. Initial work in the UK has revealed that there is reticence amongst some GP trainers to provide teaching opportunities to teach and that GP trainees themselves place low priority upon this activity given competing clinical and training assessment demands (12). Further international research is needed to explore the range of barriers that limit GP trainee involvement in teaching students in order to identify solutions to address these.

\section{Summary}

We have presented recommendations at the trainee, practice, training programme and medical school level that we believe will encourage and facilitate more teaching of medical students by GP trainees. We believe that this would benefit all those involved and in the long run, our patients. While these recommendations are largely UK-focused we believe the principles can be equally applied to any setting where community based medical education and training takes place. Further research into innovations to facilitate our recommendations in different contexts would be welcome in addition to further work on addressing identified barriers. We hope that in the near future all medical students will have had experience of 
being taught by GP trainees and likewise all GPs will qualify having had significant training and experience of teaching undergraduate students.

\section{Notes on contributors}

Hugh Alberti is Sub-dean for Primary and Community Care at Newcastle University. He also leads a team of academic GP trainees. He is a GP at Linthorpe Surgery in Middlesborough and a GP trainer.

Michael Harrison is a GP at Spring Terrace Health Centre in North Shields and an Academic Clinical Fellow at Newcastle University.

Liza Kirtchuk is a Clinical Lecturer and GP Stage 2 Lead in the King's Undergraduate Medical Education in the Community Team at King's College London. She is a GP in South London.

Joe Rosenthal is Professor of Primary Care Education at UCL Medical School, a Programme Director of the Royal Free GP Specialty Training Programme and Co-chair of the UK Heads of GP Teaching Group.

Harish Thampy is a Senior Clinical Lecturer and Academic Lead for Assessments on the MBChB Programme at the University of Manchester. He is a GP at Firsway Health Centre in Manchester and is an accredited Foundation doctor supervisor.

\section{Declaration of interests}

The authors report no declarations of interest 


\section{References}

1. Thampy H, Alberti H, Kirtchuk L, et al. Near peer teaching in general practice. $\mathrm{Br} J \mathrm{Gen}$ Pract. 2019;69(678):12-13.

2. Ramani S, Mann K, Taylor D, et al. Residents as teachers: Near peer learning in clinical work settings: AMEE Guide No. 106. Med Teach. 2016;38(7):642-655.

3. Musunuru S, Lewis B, Rikkers LF, et al. Effective Surgical Residents Strongly Influence Medical Students to Pursue Surgical Careers. J Am Coll Surg. 2007(1):164-167.

4. Whittaker LD, Estes NC, Ash J, et al. The value of resident teaching to improve student perceptions of surgery clerkships and surgical career choices. Am J Surg. 2006;191(3):320-324.

5. Johnson NR, Chen J. Medical student evaluation of teaching quality between obstetrics and gynecology residents and faculty as clinical preceptors in ambulatory gynecology. Am J Obstet Gynecol. 2006;195(5):1479-1483.

6. Ogburn JA, Espey EL, Dorin MH, et al. Obstetrics and gynecology residents as teachers of medical students: Predictors of excellence. Am J Obstet Gynecol. 2005;193(5):1831-1834.

7. General Medical Council. Good Medical Practice. 2013.

8. Royal College of General Practitioners. Royal College of General Practitioners Curriculum Statement 3.7: Teaching, Mentoring and Clinical Supervision. London: 2007.

9. Health Education England. By choice-not by chance. London: Health Education England, 2016.

10. Marshall $\mathrm{H}$, Alberti $\mathrm{H}$. Attitudes and experiences of GP trainees towards undergraduate teaching: An underused resource? Educ Prim Care. 2015;26(5):361-362.

11. Rushforth B, Kirby J, Pearson D. General practice registrars as teachers: a review of the literature. Educ Prim Care. 2010;21(4):221-229.

12. Kirby J, Rushforth B, Nagel C, et al. Should GP specialty trainees teach? Contrasting views from GP specialty trainees and their trainers. Educ Prim Care. 2014;25(2):96-102.

13. Thampy H, Agius S, Allery LA. The motivation to teach as a registrar in general practice. Educ Prim Care. 2013;24(4):244-250.

14. Alberti $\mathrm{H}$, Harrison M, Thampy H. Barriers to involving GP Speciality Trainees in the teaching of medical students in Primary Care: the GP trainer perspective. submitted Educ Prim Care.

15. Remmen R, Denekens J, Scherpbier A, et al. An evaluation study of the didactic quality of clerkships. Med Educ. 2000;34(6):460-464.

16. Williams B, Amiel C. General practice registrars as teachers: a questionnaire-based evaluation. JRSM Short Rep. 2012;3(3):14.

17. Royal College of General Practitioners. The RCGP Curriculum: 2.04 Enhancing Professional Knowledge. London: 2016.

18. General Practitioners Education and Training. A Framework for Vertical Integration in GP Education and Training. Canberra: General Practice Education and Training Ltd., 2003.

19. Dick.M.L, King.D.B, Mitchell.J.K, et al. Vertical Integration in Teaching And Learning (VITAL): an approach to medical education in general practice. Med J Aust. 2007;187(2):133-135.

20. Thistlethwaite JE, Kidd MR, Hudson JN. General practice: a leading provider of medical student education in the 21st century? Med J Aust. 2007;187(2):124-128.

21. Gray RW, Carter YH, Hull SA, et al. Characteristics of general practices involved in undergraduate medical teaching. Br J Gen Pract. 2001;51(466):371-374.

22. Gibson, Campbell. Promoting effective teaching and learning: hospital consultants identify their needs. Med Educ. 2000;34(2):126-130.

23. Halestrap P, Leeder D. GP Registrars As Teachers: A survey of Their Level of Involvement and Training. Educ Prim Care. 2011;22(5):310-313 
24. Hill AG, Yu T-C, Barrow M, et al. A systematic review of resident-as-teacher programmes. Med Educ. 2009;43(12):1129-1140.

25. Steinert $\mathrm{Y}, \mathrm{Mann} \mathrm{K}$, Anderson B, et al. A systematic review of faculty development initiatives designed to enhance teaching effectiveness: A 10-year update: BEME Guide No. 40. Med Teach. 2016;38(8):769-786

26. Marshall $\mathrm{H}$, Alberti $\mathrm{H}$, Cope $\mathrm{S}$, et al. Nurturing future academics and leaders: extended integrated training posts in general practice. BMJ. 2017;356:1007.

27. Cope S, Alberti H. Exploring the Experience of Undertaking an Educational Integrated Training Post in General Practice. Masters dissertation. 2018.

28. Gusic M, Hageman H, Zenni E. Peer review: a tool to enhance clinical teaching. Clin Teach. 2013;10(5):287-290. 